\title{
Adição de farinha da casca de melão em cupcakes altera a composição físico-química e a aceitabilidade entre crianças
}

\author{
Addition of melon peel flour in cupcakes alter physico-chemical \\ composition and children acceptability
}

Rafaely de Fátima Fernandes Almeida Vieira ${ }^{1}$, Cláudio Luís da Silva Carvalho², Izabella Renatta Almeida de Carvalho², Camila Jordão Candido², Elisvânia Freitas dos Santos ${ }^{2}$, Daiana Novello ${ }^{1}$

${ }^{1}$ Universidade Estadual do Centro-Oeste (UNICENTRO), Guarapuava, Paraná, Brasil.

${ }^{2}$ Universidade Federal de Mato Grosso do Sul (UFMS), Campo Grande, Mato Grosso do Sul, Brasil.

\begin{abstract}
Resumo
Introdução: O desperdício de alimentos apresenta números alarmantes em todo o mundo. As cascas de frutas se destacam entre os subprodutos que mais são descartados pela população. Dessa forma, são necessárias estratégias que visem a aproveitar os alimentos de uma forma integral, com o intuito de combater a fome e o impacto ambiental do descarte de resíduos. Objetivo: Avaliar a aceitabilidade sensorial de cupcakes adicionados de diferentes níveis de farinha da casca de melão (FCM), entre crianças. Também, determinar a composição físico-química do produto padrão e daquele com maior teor de FCM e aceitação semelhante ao padrão. Metodologia: Foram desenvolvidas cinco formulações de cupcakes: F1 (padrão - 0\% de FCM) e as demais adicionadas de 4,25\% (F2), 8,5\% (F3), 12,75\% (F4) e $17 \%$ (F5) de FCM. Participaram da avaliação sensorial 60 provadores não treinados, de ambos os gêneros, com idade entre 7 e 10 anos. Resultados: Maiores notas foram verificadas para F1 comparada à F5 para os atributos de aparência, aroma, sabor e aceitação global $(\mathrm{p}<0,05)$. Para os atributos de textura, cor e para a intenção de compra não houve diferença estatística entre os produtos $(p>0,05)$. Maiores teores de umidade $(\mathrm{p}<0,05)$ e menores de cinzas e fibras foram constatados em $\mathrm{F} 1$, quando comparada à F4. Conclusão: Um nível de adição de até $12,75 \%$ de FCM em cupcakes foi bem aceito pelos provadores infantis, obtendo-se aceitação sensorial semelhante ao produto padrão e com boas expectativas de comercialização.
\end{abstract}

Palavras-chave: Aceitabilidade; Subprodutos; Nutrientes.

Autor correspondente:

Daiana Novello

Endereço: Rua Simeão Camargo Varela de Sá, 03, Vila Carli

Recebido em: 03/06/2017

CEP: 85040-080, Guarapuava, Paraná, Brasil.

E-mail: nutridai@gmail.com

Revisado em: 07/08/2017

Aceito em: 22/08/2017

Publicado em: 31/08/2017 


\section{Abstract}

Introduction: Food waste presents alarming numbers worldwide. The fruit peels stand out among the by-products that are most discarded by the population. In this way, strategies are needed to take advantage of food in an integral way, in order to combat hunger and the environmental impact of waste disposal. Objective: To evaluate the sensory acceptability of added cupcakes of different levels of melon peel flour (MPF) among children. Also, determine the physico-chemical composition of the standard product and the one with the highest content of MPF and acceptance similar to the standard. Methodology: Five formulations of cupcakes were developed: F1 (standard - 0\% MPF) and the others added of $4.25 \%(F 2), 8.5 \%$ (F3), $12.75 \%$ (F4) and 17\% (F5) of MPF. Sixty untrained testers of both genders, aged between 7 and 10 years, participated in the sensory evaluation. Results: Higher scores were observed for F1 compared to F5 for the appearance, aroma, taste and overall acceptance attributes $(p<0.05)$. For the attributes of texture, color and for the purchase intention there was no statistical difference between the products $(p>0.05)$. Higher moisture contents $(p<0.05)$ and lower ash and fiber contents were observed in F1, when compared to F4. Conclusion: A level of addition of up to $12.75 \%$ of $M P F$ in cupcakes was well accepted by the children's tasters, obtaining sensory acceptance similar to the standard product and with good marketing expectations.

Keywords: Acceptability; Byproducts; Nutrients.

\section{Introdução}

No mundo, cerca de 1,3 bilhões de toneladas de alimentos são desperdiçadas a cada ano. Com isso, verificam-se prejuízos econômicos, além de gerar um impacto significativo sobre a sobrevivência alimentar da população ${ }^{1}$. O Brasil descarta 39 milhões de toneladas de produtos alimentícios diariamente ${ }^{2}$. Isso, porque apresenta uma elevada produção de hortifrutigranjeiros (87,5 milhões de toneladas, aproximadamente) $)^{3,4}$, os quais representam a maior parte dos produtos descartados. O desperdício de alimentos inicia na fase de plantio, percorrendo durante a colheita, armazenamento, transporte e, também, na mesa do consumidor final ${ }^{5}$. Nesses casos, verifica-se que as cascas, talos, sementes, folhas e outras partes consideradas não convencionais são geralmente desprezadas. Contudo, sabe-se que a utilização desses subprodutos em diferentes preparações poderia melhorar o teor nutricional dos alimentos, já que muitas vezes apresentam um elevado conteúdo de vitaminas e minerais. Visando reduzir o desperdício alimentar, muitos estudos já avaliaram a adição de subprodutos, como a casca de banana, abacaxi, maracujá e melão, em doces, bolos, sucos, geleia e saladas, obtendo boa aceitabilidade sensorial ${ }^{6-}$ 9 .

O melão (Cucumis melo L.) é uma fruta tropical de formato oval. O sabor é adocicado, podendo apresentar coloração de casca amarela, branca e verde. A polpa pode ser de diferentes cores como branca, amarela, laranja, salmão e verde-claro. O melão mais comum e de maior preferência são aquelas com casca amarela $^{10-11}$. A fruta tem origem asiática e africana. É cultivada em grande escala no Brasil, principalmente na região nordeste que apresenta clima tropical e semiárido $^{12}$. Em 2015, a produção brasileira de melão foi de aproximadamente 589.939 toneladas, foram exportados $223.746 .193 \mathrm{~kg}^{13}$, o que demonstra sua grande importância comercial. Geralmente, o melão é consumido na forma in natura ou na forma de sucos, compotas e produtos de confeitaria ${ }^{14}$. Apresenta um bom perfil nutricional, com elevados teores de carboidratos $\left(92,8\right.$ g. $\left.100 \mathrm{~g}^{-1}\right)$, vitamina A (116 mcg. $\left.100 \mathrm{~g}^{-1}\right)$ e vitamina C $\left(29 \mathrm{mg} \cdot 100 \mathrm{~g}^{-1}\right)^{15}$. No entanto, verifica-se um grande desperdício desse alimento, que ocorre principalmente devido ao armazenamento inadequado, curto shelf-life, despadronização de cor e formato, dentre outros. A casca do melão é, quase sempre, descartada, já que os consumidores desconhecem sua possível utilização como ingrediente em diferentes produtos. Com isso, seria possível aumentar a diversidade alimentar entre populações mais vulneráveis como as crianças.

A fase escolar compreende o período da vida entre os sete e dez anos. Em geral, essa etapa pode influenciar novos hábitos de vida, já que a escola é considerada como um local ideal para melhorar e agregar novos conhecimentos. As crianças tendem a adquirir certa autonomia nas escolhas alimentares, podendo ocorrer alguma interferência de colegas, professores e outros agentes para o consumo de alimentos. Assim, ações voltadas à correção de hábitos alimentares ainda na infância podem colaborar para promover e recuperar a saúde, por meio de uma alimentação mais saudável nas demais fases da vida ${ }^{16}$. Como resultado espera-se reduzir as chances do desenvolvimento de doenças crônicas não 
transmissíveis futuras $(\mathrm{DCNT})^{17}$. Crianças em fase escolar geralmente apresentam um baixo consumo de frutas e hortaliças e elevada ingestão de açúcares, sódio e gorduras ${ }^{18}$. Além disso, é comum ocorrer uma condição conhecida como neofobia alimentar, a qual é caracterizada pela rejeição a novos alimentos ${ }^{18}$. Algumas estratégias educativas podem ser utilizadas para modificar esse cenário. Um exemplo é a disponibilização de alimentos saudáveis em ambientes comuns às crianças, como as escolas, o que pode incentivar a ingestão de alimentos que apresentem um melhor perfil nutricional, contendo elevados teores de fibras, vitaminas e minerais ${ }^{17}$.

Os cupcakes são conhecidos como bolos em porções individuais. São obtidos a partir da combinação de farinha (s) ou fécula (s), acrescidas de outros ingredientes, fermentados $\mathrm{e}$ assados. Os cupcakes podem ser decorados, recheados ou simples e apresentar diferentes texturas ${ }^{19}$. Entre os anos de 2008 e 2012, o mercado brasileiro de bolos mostrou crescimento de 57\%, a seção de cupcakes foi responsável por $66,8 \%$ das vendas. No ano de 2015 , o mercado de bolos industriais no Brasil atingiu 0,18 $\mathrm{kg} / \mathrm{ano}$ com 37,3 mil toneladas e faturamento de 0,85 bilhões de reais ${ }^{20}$. Os cupcakes apresentam praticidade de fabricação, além de serem aceitos pelos mais diversos públicos, com destaque para as crianças ${ }^{21}$. Apesar disso, podem conter elevados teores de açúcares $\left(40\right.$ g. $\left.100 \mathrm{~g}^{-1}\right)$ e gorduras $\left(12 \mathrm{~g} \cdot 100 \mathrm{~g}^{-1}\right)^{22}$. Considerando esse aspecto, novas estratégias tecnológicas podem ser desenvolvidas visando melhorar o perfil nutricional dos cupcakes, já que apresentam evado potencial para a adição de ingredientes mais saudáveis.

Para a comercialização de um novo produto, fazse necessário a utilização de testes que permitam avaliar principalmente as características físicoquímicas e sensoriais. Os testes de aceitabilidade sensorial têm o objetivo de definir a qualidade sensorial do produto, e são considerados eficazes para o desenvolvimento de novas alternativas alimentares ${ }^{19}$. Esses testes têm sido amplamente aplicados para o público infantil, uma vez que, atualmente, as crianças possuem grande influência na aquisição de produtos $^{23}$. Por outro lado, as análises físico-químicas são importantes para avaliar se os alimentos estão adequados para o consumo e posterior comercialização, garantindo a saúde do consumidor ${ }^{24}$.

O objetivo deste trabalho foi avaliar a aceitabilidade sensorial de cupcakes adicionados de diferentes níveis de farinha da casca de melão (FCM), entre crianças. Também, determinar a composição físico-química do produto padrão e daquele com maior teor de FCM e aceitação semelhante ao padrão.

\section{Metodologia}

\section{Aquisição da matéria-prima}

Os ingredientes foram adquiridos em supermercados do município de Guarapuava, PR.
Foram utilizados melões amarelos, com bom aspecto visual, sem imperfeições e de tamanho médio.

\section{Preparo da farinha da casca de melão}

Inicialmente, $50,33 \mathrm{~kg}$ de melão foram higienizados em água corrente potável, sanitizados (1 litro de água para cada $8 \mathrm{ml}$ de água sanitária, por 10 minutos), e novamente higienizados em água corrente potável. Em seguida, as cascas foram extraídas de forma manual (1,5 $\mathrm{mm}$ espessura) com o auxílio de uma faca, e picadas em tamanho aproximado de $5 \mathrm{~cm}$, obtendo rendimento de $8 \mathrm{~kg}$. As cascas foram acomodadas em telas (treliça/32 mesh) e submetidas à secagem em estufa (Pardal ${ }^{\circledR}$, Brasil), com circulação de ar $\left(70^{\circ} \mathrm{C}\right)$ por 48 horas. Depois de desidratadas, permaneceram em temperatura ambiente $\left(22^{\circ} \mathrm{C}\right)$ até total resfriamento. Para o preparo da farinha, as cascas secas foram trituradas em liquidificador doméstico (Britânia ${ }^{\circledR}$, Brasil) e passadas em peneira com abertura de $0,5 \mathrm{~mm}$ (Bertel ${ }^{\circledR}$, Brasil). A FCM obteve um rendimento de $705 \mathrm{~g}$.

\section{Formulações}

As formulações foram preparadas individualmente no Laboratório de Técnica Dietética do Departamento de Nutrição da Universidade Estadual do Centro-Oeste (UNICENTRO). Foram elaboradas 5 formulações de cupcakes: $\mathrm{F} 1$ padrão $(0 \%$ de FCM) e as demais adicionadas de $4,25 \%$ (F2), $8,5 \%$ (F3), $12,75 \%(\mathrm{~F} 4)$ e $17 \%$ (F5) de FCM. Esses níveis de adição foram definidos por meio de testes sensoriais preliminares realizados com o produto. Além das porcentagens de FCM, os seguintes ingredientes foram utilizados nas formulações: farinha de trigo (F1: 19\%, F2: $14,75 \%$, F3: 10,5\%, F4: 6,25\% e F5: 2\%), açúcar refinado $(20,21 \%)$, leite integral $(18,60 \%)$, ovos $(17,18 \%)$, semente de linhaça $(12,18 \%)$, manteiga sem sal $(11,62 \%)$, essência de baunilha $(0,51 \%)$, fermento em pó $(0,51 \%)$ e sal $(0,20 \%)$. Os ingredientes manteiga e açúcar foram misturados manualmente até formar uma massa homogênea. Em seguida, foram adicionados os ovos, a essência de baunilha, a farinha de trigo, a FCM, o fermento em pó e o sal. Por último, foram acrescidos o leite e a linhaça, misturados até total homogeneização. As formulações foram dispostas em formas individuais de papel específicas para cupcake ( $5 \mathrm{~cm}$ de diâmetro), e acomodadas em formas de alumínio. Os cupcakes foram assados em forno convencional (Atlas ${ }^{\circledR}$, Brasil) pré-aquecido (180 ${ }^{\circ} \mathrm{C}$ ), por aproximadamente 30 minutos. Na sequência, permaneceram em temperatura ambiente $\left(22^{\circ} \mathrm{C}\right)$ para resfriamento. As formulações foram armazenadas em potes plásticos hermeticamente fechados até a realização das análises.

\section{Análise sensorial}

Participaram dos testes sensoriais 60 crianças, não treinadas, devidamente matriculadas em uma Escola Municipal de Guarapuava, PR, de ambos os gêneros, com idade entre 7 a 10 anos. Cada prova foi feita individualmente em uma sala da escola. O provador foi orientado pelas pesquisadoras para o preenchimento das respostas. Foram avaliados os 
atributos de aparência, aroma, sabor, textura e cor, por meio de uma escala hedônica facial estruturada mista de 7 pontos variando de 1 ("super ruim") a 7 ("super bom"). Também, foram aplicadas questões de aceitação global e intenção de compra analisadas com uma escala estruturada de 5 pontos (1 - "desgostei muito"/"não compraria" a 5 - "gostei muito"/"compraria com certeza") ${ }^{25}$. Os provadores receberam uma porção de cada amostra (aproximadamente $10 \mathrm{~g}$ ), em copos descartáveis brancos $(50 \mathrm{ml})$, codificados com números de três dígitos, de forma casualizada e balanceada. Também receberam um copo de água para limpeza do palato. As formulações foram oferecidas aos provadores de forma monádica sequencial.

O teste de comparação múltipla foi aplicado para comparar as amostras de cupcakes elaboradas com um produto similar (tradicional) comercializado no mercado (referência). Cada provador identificou se as formulações apresentavam sabor melhor, igual ou pior ao produto referência em uma escala hedônica estruturada de 7 pontos, variando da nota 1 ("extremamente pior que o primeiro") a nota 7 ("extremamente melhor que o primeiro") 25 . O cálculo do índice de aceitabilidade (IA) foi realizado segundo a fórmula: IA $(\%)=\mathrm{A} \times 100 / \mathrm{B}(\mathrm{A}=$ nota média obtida para o produto; $\mathrm{B}=$ nota máxima dada ao produto $)^{25}$.

\section{Composição físico-química}

As seguintes determinações foram realizadas em triplicata na FCB, na formulação padrão e naquela com maior teor de FCB e com aceitação sensorial semelhante a padrão: Umidade: determinada em estufa a $105^{\circ} \mathrm{C}$ até peso constante ${ }^{26}$; Cinzas: analisadas em mufla $\left(550^{\circ} \mathrm{C}\right)^{26}$; Lipídios totais: utilizou-se o método de extração a quente com extrator de Soxhlet e éter de petróleo ${ }^{26}$; Proteínas: avaliadas através do teor de nitrogênio total da amostra, pelo método Kjeldahl, determinado ao nível semimicro ${ }^{26}$. Utilizou-se o fator de conversão de nitrogênio para proteína de 6,25; Fibra Alimentar: mensurada por cálculo teórico ${ }^{27-28}$;
Carboidratos: avaliados por meio de cálculo teórico (por diferença) nos resultados das triplicatas, conforme a fórmula: \% Carboidratos $=100-(\%$ umidade $+\%$ proteína $+\%$ lipídios $+\%$ cinzas $+\%$ fibra alimentar); Valor calórico total (kcal): calculado teoricamente utilizando-se os seguintes valores: lipídios $(8,37 \mathrm{kcal} / \mathrm{g})$, proteína $(3,87 \mathrm{kcal} / \mathrm{g})$ e carboidratos $(4,11 \mathrm{kcal} / \mathrm{g})^{29}$.

\section{Determinação do valor diário de referência (VD)}

$\mathrm{O}$ VD foi calculado em relação a $50 \mathrm{~g}$ da amostra, com base nos valores médios, preconizados para crianças de 7 a 10 anos $^{30}$, resultando em: 1.818 $\mathrm{kcal} / \mathrm{dia}, 248,05 \mathrm{~g} / \mathrm{dia}$ de carboidratos, 63,47 g/dia de proteínas, 66,53 g/dia de lipídios e 12,38 g/dia de fibra alimentar.

\section{Análise estatística}

Os dados foram analisados com auxílio do software Statgraphics Plus ${ }^{\circledR}$, versão 5.1, por meio da análise de variância (ANOVA). A comparação de médias foi realizada pelo teste de médias de Tukey, $\mathrm{t}$ de student e Dunnett, avaliados com nível de 5\% de significância.

\section{Questões éticas}

Este trabalho foi aprovado pelo Comitê de Ética em Pesquisa da UNICENTRO, parecer número $\mathrm{n}^{\circ}$ 608.950/2014. Os critérios de exclusão foram: possuir alergia a algum ingrediente utilizado na elaboração dos cupcakes, não ser aluno da escola em questão ou não entregar o Termo de Consentimento Livre e Esclarecido (TCLE) assinado pelo responsável legal.

\section{Resultado e discussão}

\section{Análise sensorial}

Por meio da Tabela 1 verificam-se os resultados da avaliação sensorial do cupcake padrão e adicionados de FCM.

TABELA 1: Resultados do índice de aceitabilidade e dos testes de aceitação e de intenção de compra dos cupcakes com adição de farinha da casca de melão

\begin{tabular}{|c|c|c|c|c|c|}
\hline Formulações/ & F1 & F2 & F3 & F4 & F5 \\
\hline Atributos & Média $\pm E P M$ & Média $\pm E P M$ & Média $\pm E P M$ & Média $\pm E P M$ & Média $\pm E P M$ \\
\hline Aparência & $6,29 \pm 0,12^{\mathrm{a}}$ & $6,12 \pm 0,14^{\mathrm{ab}}$ & $5,90 \pm 0,14^{\mathrm{ab}}$ & $5,76 \pm 0,13^{\mathrm{ab}}$ & $5,61 \pm 0,06^{\mathrm{b}}$ \\
\hline $\mathrm{IA}(\%)$ & 89,86 & 87,43 & 84,29 & 82,29 & 80,14 \\
\hline Aroma & $6,26 \pm 0,12^{\mathrm{a}}$ & $6,21 \pm 0,14^{\mathrm{ab}}$ & $6,00 \pm 0,14^{\mathrm{ab}}$ & $5,90 \pm 0,15^{\mathrm{ab}}$ & $5,65 \pm 0,2^{\mathrm{b}}$ \\
\hline IA $(\%)$ & 89,43 & 88,71 & 85,71 & 84,30 & 80,71 \\
\hline Sabor & $6,46 \pm 0,12^{\mathrm{a}}$ & $6,38 \pm 0,14^{\mathrm{a}}$ & $6,26 \pm 0,16^{\mathrm{a}}$ & $6,03 \pm 0,15^{\mathrm{ab}}$ & $5,62 \pm 0,22^{\mathrm{b}}$ \\
\hline IA $(\%)$ & 92,30 & 91,14 & 89,43 & 86,14 & 80,30 \\
\hline Textura & $6,13 \pm 0,14^{\mathrm{a}}$ & $6,10 \pm 0,14^{\mathrm{a}}$ & $6,05 \pm 0,13^{\mathrm{a}}$ & $5,80 \pm 0,14^{\mathrm{a}}$ & $5,64 \pm 0,16^{\mathrm{a}}$ \\
\hline IA $(\%)$ & 87,57 & 87,14 & 86,43 & 82,86 & 80,57 \\
\hline Cor & $5,92 \pm 0,14^{\mathrm{a}}$ & $5,91 \pm 0,11^{\mathrm{a}}$ & $5,84 \pm 0,13^{\mathrm{a}}$ & $5,86 \pm 0,15^{\mathrm{a}}$ & $5,82 \pm 0,16^{\mathrm{a}}$ \\
\hline $\mathrm{IA}(\%)$ & 84,57 & 84,23 & 83,43 & 83,71 & 83,14 \\
\hline Aceitação global & $4,63 \pm 0,07^{\mathrm{a}}$ & $4,65 \pm 0,08^{\mathrm{a}}$ & $4,53 \pm 0,10^{\mathrm{ab}}$ & $4,52 \pm 0,10^{\mathrm{ab}}$ & $4,18 \pm 0,04^{b}$ \\
\hline IA $(\%)$ & 92,60 & 93,00 & 90,60 & 90,40 & 83,60 \\
\hline
\end{tabular}


Adlição de farinha da casca de melão em cupcakes

Intenção de compra

$4,62 \pm 0,90^{\mathrm{a}}$

$4,52 \pm 0,11^{\mathrm{a}}$

$4,38 \pm 0,14^{\mathrm{a}}$

$4,37 \pm 0,14^{\mathrm{a}}$

$4,33 \pm 0,16^{\mathrm{a}}$

IA (\%)

92,40

90,40

87,60

87,40

86,60

Letras diferentes na linha indicam diferença significativa pelo teste de Tukey $(\mathrm{p}<0,05)$; EPM: erro padrão da média; IA: índice de aceitabilidade. Adição de farinha da casca de melão: F1: 0\%; F2: 4,25\%; F3: 8,5\%; F4: 12,75\%; F5: $17 \%$. Fonte: os autores.

Maiores notas foram verificadas para F1 comparada à F5 para os atributos de aparência e aroma $(\mathrm{p}<0,05)$, sem diferença estatística entre as demais amostras. Resultados similares para o aroma foram verificados por Miranda et al. ${ }^{31}$, que avaliaram bolos enriquecidos com farinha da casca de maracujá $(0,7 \%$, 0\% e 14\%), entre adultos. Os ácidos fenólicos, flavonas, açúcares e ácidos orgânicos presentes na casca de melão contribuem para a alteração do $\operatorname{aroma}^{28}$ e, consequentemente, para as menores notas de F5.

As formulações F1, F2 e F3 apresentaram maior aceitabilidade ( $\mathrm{p}<0,05)$ que F5 quanto ao sabor, já F4 não diferiu significativamente das demais amostras. Dados que corroboram com Carvalho et al. ${ }^{19}$, que estudaram cupcakes adicionados de farinha da casca de banana $(0,2,5 \%, 4 \%, 5,5 \%$ e $7 \%)$, entre adultos. $\mathrm{O}$ aquecimento dos compostos fenólicos, presentes em elevadas quantidades em cascas de frutas, prejudica o sabor, promovendo certa adstringência ${ }^{32}$, o que, possivelmente, desencadeia uma menor aceitação em produtos adicionados desses subprodutos.

Para os atributos de textura, cor e para a intenção de compra não houve diferença estatística entre os produtos $(p>0,05)$. Efeito similar ao verificado por Vizu et al. ${ }^{6}$ para cor, que avaliaram doces em pasta adicionados de farinha da casca de banana $(0 \%$ e $10 \%$ ), entre em adultos. Apesar disso, quando se adiciona farinha da casca de melão em produtos alimentícios, a cor é o primeiro atributo a ser rejeitado pelo consumidor ${ }^{28}$. Isso, porque compostos presentes na casca como a clorofila, os carotenoides e os flavonoides interferem na cor, já que agem como

corantes naturais em frutas e hortaliças ${ }^{33}$. Maiores notas para aceitação global foram referidas para F1 e $\mathrm{F}$ 2, comparadas à F5. As demais amostras não foram diferentes estatisticamente $(\mathrm{p}>0,05)$.

Durante a preparação dos cupcakes, verificou-se que as formulações com maior quantidade de FCM tornaram-se mais quebradiças. Isso porque nessas amostras havia uma menor quantidade de glúten, devido à redução nos teores de farinha de trigo. Sabese que o glúten, formado pela combinação da água com as proteínas gluteína e gliadina, apresenta funções tecnológicas importantes em produtos alimentícios, como intensificador de textura, espessante, aromatizante e levedante ${ }^{34}$. Nesse aspecto, compreende-se que o volume dos cupcakes adicionados de FCM foi influenciado pela redução da capacidade de formação do complexo gluteína e gliadina, que diminui a expansão e retenção dos gases pela massa ${ }^{35}$.

Todas as amostras tiveram IA's maiores que $70 \%$, classificando os cupcakes com boa aceitabilidade sensorial $^{36}$. Os resultados favoráveis apresentados na pesquisa podem colaborar para uma maior ingestão de fibras pelas crianças, já que esse público apresenta, geralmente, um baixo consumo desse componente ${ }^{37}$. Além disso, a literatura demonstra que um consumo adequado de fibras está associado à redução do risco de DCNT como a diabetes mellitus, as hipertrigliceridemias, dentre outras ${ }^{38}$. Na Figura 1, está descrita a distribuição dos provadores pelos valores hedônicos avaliados no teste sensorial dos cupcakes adicionados de FCM.

FIGURA 1: Distribuição dos provadores pelos valores hedônicos obtidos na avaliação dos cupcakes. Adição de farinha da casca de melão: F1: 0\%; F2: 4,25\%; F3: 8,5\%; F4: 12,75\%; F5: 17\%. Fonte: os autores.
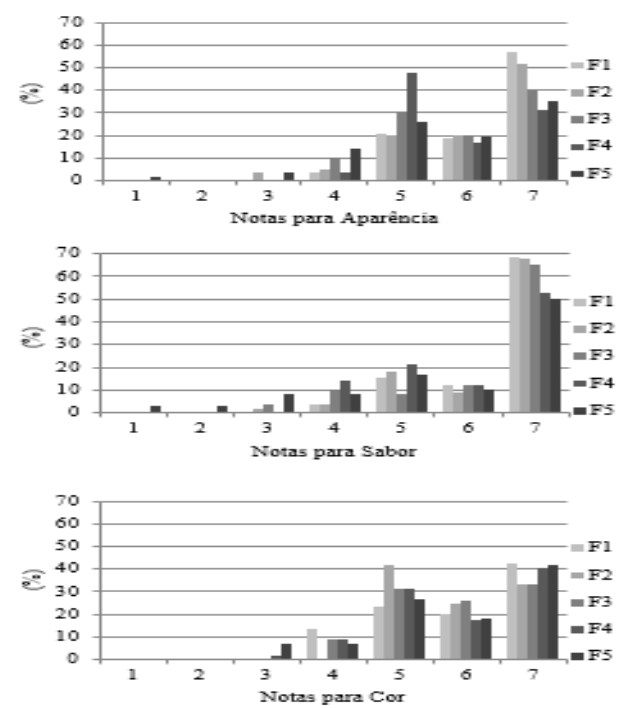
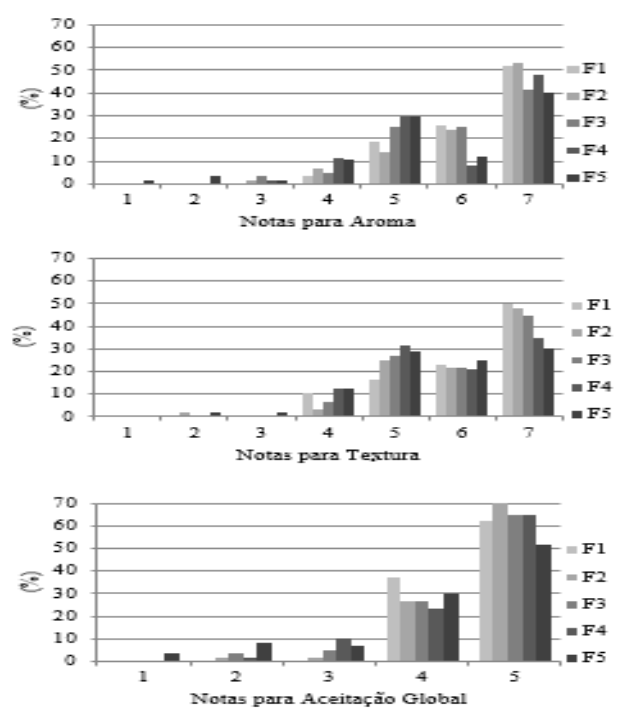
A maioria das notas conferidas pelos provadores encontram-se acima de 5 (bom) para os atributos e 4 (gostei) para aceitação global, o que demonstra que as formulações foram, em geral, bem aceitas pelas crianças. Na Figura 2, estão apresentados os resultados do teste de comparação múltipla das amostras elaboradas comparadas com um cupcake tradicional comercializado (referência).

FIGURA 2: Médias do teste de comparação múltipla (sabor) dos cupcakes comparados a um produto comercial (referência). *Diferença significativa do produto comercial ( $\mathrm{p}<0,05$; teste de Dunnett). Adição de farinha da casca de melão: F1: 0\%; F2: 4,25\%; F3: 8,5\%; F4: 12,75\%; F5: 17\%. Fonte: os autores.

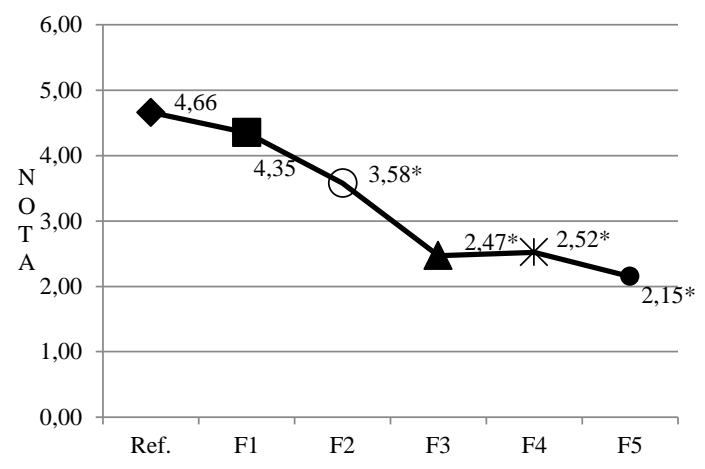

As formulações F2, F3, F4 e F5 apresentaram sabor inferior ao cupcake comercializado, enquanto a amostra padrão foi considerada similar. Contudo, considerando que a amostra F4 foi aquela com maior teor de FCM e com aceitação sensorial similar a padrão em todas as avaliações realizadas (Tabela 1), a mesma foi selecionada para fins de comparação físicoquímica juntamente com a formulação padrão (F1).

\section{Composição físico-química}

A composição físico-química e os valores diários recomendados do cupcake padrão e daquele acrescido de $12,75 \%$ de FCM estão apresentados na Tabela 2.

TABELA 2: Média da composição físico-química da farinha da casca de melão e das formulações de cupcake F1 e F4

\begin{tabular}{|c|c|c|c|c|c|}
\hline & FCM & F1 & & F4 & \\
\hline Avaliação & Média $\pm D P$ & Média $\pm D P$ & $\operatorname{VD}(\%)^{*}$ & Média $\pm D P$ & $\operatorname{VD}(\%)^{*}$ \\
\hline Umidade $\left(\mathrm{g} .100 \mathrm{~g}^{-1}\right)$ & $9,86 \pm 0,04$ & $28,12 \pm 0,08^{\mathrm{a}}$ & $\mathrm{ND}$ & $26,77 \pm 0,04^{\mathrm{b}}$ & ND \\
\hline Cinzas $\left(\mathrm{g} .100 \mathrm{~g}^{-1}\right)$ & $2,16 \pm 0,03$ & $1,37 \pm 0,03^{\mathrm{b}}$ & ND & $2,52 \pm 0,02^{\mathrm{a}}$ & ND \\
\hline Proteínas (g.100g $\left.\mathrm{g}^{-1}\right)$ & $10,84 \pm 0,08$ & $7,20 \pm 0,05^{\mathrm{a}}$ & 5,67 & $7,04 \pm 0,06^{\mathrm{a}}$ & 5,54 \\
\hline Lipídios (g.100g-1) & $1,65 \pm 0,08$ & $18,10 \pm 0,05^{\mathrm{a}}$ & 13,60 & $18,14 \pm 0,04^{\mathrm{a}}$ & 13,63 \\
\hline Carboidratos (g.100g $\left.\mathrm{g}^{-1}\right)$ & $75,48 \pm 0,15$ & $45,22 \pm 0,25^{\mathrm{a}}$ & 9,12 & $45,53 \pm 0,17^{\mathrm{a}}$ & 9,18 \\
\hline Calorias (kcal.100g-1) & $366,01 \pm 0,65$ & $365,19 \pm 0,78^{\mathrm{a}}$ & 10,04 & $366,21 \pm 0,98^{\mathrm{a}}$ & 10,07 \\
\hline Fibra alimentar $\left(\mathrm{g} .100 \mathrm{~g}^{1}\right)^{* *}$ & 34,62 & 4,52 & 18,25 & 8,63 & 69,70 \\
\hline
\end{tabular}

Letras distintas na linha indicam diferença significativa pelo teste de t de student ( $<<0,05)$; FCM: farinha da casca de melão. Adição de FCM: F1: 0\%; F4: 12,75\%; *Valor diário de referência (VD): nutrientes avaliados pela média da DRI ${ }^{30}$, com base numa dieta de $1.818 \mathrm{kcal} /$ dia e porção média de $50 \mathrm{~g}$; Valores calculados em base úmida; **Cálculo teórico $27-$ 28. DP: desvio padrão da média; ND: não disponível. Fonte: os autores. 
Valores inferiores ao presente trabalho para a FCM foram verificados pela literatura ${ }^{28}$ para proteínas $\left(6,21 \quad\right.$ g. $\left.100 \mathrm{~g}^{-1}\right)$ e carboidratos $\left(57,94 \quad\right.$ g. $\left.100 \mathrm{~g}^{-1}\right)$, superiores para umidade $\left(16,95 \mathrm{~g} \cdot 100 \mathrm{~g}^{-1}\right)$, cinzas $(3,04$ g. $\left.100 \mathrm{~g}^{-1}\right)$ e similar para lipídios $\left(1,76 \mathrm{~g} .100 \mathrm{~g}^{-1}\right)$. As técnicas empregadas no cultivo, a espessura das cascas e o binômio tempo-temperatura referentes ao processo de obtenção da farinha podem explicar as diferenças entre esses resultados ${ }^{39-40}$. $\mathrm{O}$ teor de cinzas presente na FCM demonstra a elevada concentração de minerais na casca de melão, principalmente, de cálcio $(483,7$ $\left.\mathrm{mg} / 100 \mathrm{~g}^{-1}\right)$, potássio $\left(2.046,5 \mathrm{mg} / 100 \mathrm{~g}^{-1}\right)$ e sódio $\left(491,2 \mathrm{mg} / 100 \mathrm{~g}^{-1}\right)^{41}$. Desse modo, a FCM colabora para melhorar o valor nutricional das preparações. Além disso, contém um elevado teor de fibra, que auxilia no trânsito intestinal, reduz o risco de obesidade e colabora no fortalecimento do sistema imunológico ${ }^{42}$. A FCM apresentou teor de umidade conforme o recomendado (até $15 \%$ ) pela $\mathrm{RDC} \mathrm{n}^{\circ} 263$, referente à regulamentação de Produtos de Cereais, Amidos, Farinhas e Farelos ${ }^{43}$. As farinhas em geral não podem ser consideradas fontes proteicas, contudo apresentam baixo teor de lipídeos ${ }^{4-45}$.

Maiores teores de umidade $(p<0,05)$ foram constatadas em F1, o que se deve a maior presença de água na farinha de trigo $\left(13,0 \mathrm{~g} \cdot 100 \mathrm{~g}^{-1}\right)^{27}$, quando comparada à FCM (Tabela 2). O teor de umidade verificado em F1 e F4 encontra-se conforme a recomendação da literatura para bolos $(30 \%)^{46}$. Verificou-se um maior teor de cinzas em F3, já que a farinha de trigo contém menor quantidade de minerais em sua composição $\left(0,8 \mathrm{~g} \cdot 100 \mathrm{~g}^{-1}\right)^{27}$, comparada à FCM. Não houve diferença significativa $(p<0,05)$ entre os teores de proteínas, lipídios, carboidratos e calorias nas formulações, já que o conteúdo desses nutrientes é similar entre a farinha de trigo $\left(9,8 \mathrm{~g} \cdot 100 \mathrm{~g}^{-}\right.$ ${ }^{1} ; 1,4$ g. $100 \mathrm{~g}^{-1} ; 75,1$ g. $100 \mathrm{~g}^{-1} ; 360$ kcal.100g ${ }^{-1}$, respectivamente) e a FCM. Ressalta-se que o teor de fibras verificado na formulação adicionada de $12,75 \%$ de FCM (F4), expressa um aumento significativo de $91 \%$ em relação a F1. Isso se deve, principalmente, ao elevado teor de fibras presente na FCM $\left(34,62\right.$ g. $100 \mathrm{~g}^{-}$ $\left.{ }^{1}\right)^{28}$, bem superior ao encontrado na farinha de trigo $\left(2,3 \mathrm{~g} \cdot 100 \mathrm{~g}^{-1}\right)^{27}$. Nesse aspecto, a amostra F4 pode ser considerada como um produto com alto teor de fibra alimentar, já que possui um teor mínimo de $6 \%$ de fibras em sua composição ${ }^{47}$.

\section{Conclusão}

Um nível de adição de até $12,75 \%$ de farinha da casca melão em cupcakes é bem aceito pelas crianças, obtendo-se aceitação sensorial semelhante ao produto padrão. Além disso, proporcionou um aumento no aporte de cinzas e fibras, melhorando o perfil nutricional do produto. Dessa forma, a farinha da casca de melão pode ser considerada um potencial ingrediente para adição em cupcakes e similares, com possibilidade de ser oferecida ao público infantil e com altas expectativas de comercialização.

\section{Declaração de conflitos de interesses}

Os autores do artigo afirmam que não houve nenhuma situação de conflito de interesse, tais como propostas de financiamento, emissão de pareceres, promoções ou participação em comitês consultivos ou diretivos, entre outras, que pudessem influenciar no desenvolvimento do trabalho.

\section{Referências}

1. FOOD AND AGRICULTURE ORGANIZATION (FAO). Food waste harms climate, water, land and biodiversity - new FAO report. Disponível em: $<$ http://www.fao.org/ news/story/en/item/196220/icode/>. Acesso em: 05 de junho de 2017.

2. SILVÉRIO, G. A.; OLTRAMARI, K. Desperdício de alimentos em Unidades de Alimentação e Nutrição brasileiras. Ambiência, Guarapuava, v.10, n.1, p.125133, 2014.

3. SECRETARIA DA AGRICULTURA E DO ABASTECIMENTO DO PARANÁ (SEAB). Fruticultura. Paraná, 2015. Disponível em: $<$ http://www.agricultura.pr.gov.br/arquivos/File /deral/Prognosticos/fruticultura_2014_15.pdf >. Acesso em: 02 de maio de 2017.

4. SEBRAE NACIONAL. O mercado de hortaliças no Brasil. Brasília, 2015. Disponível em: <https://www.sebrae.com.br/sites/PortalSebrae/artigo s/o-mercado-de-hortalicas-no-brasil,92e 8634e2ca62410VgnVCM100000b272010aRCRD>. Acesso em: 04 de maio de 2017.

5. SOARES, A. G. Desperdício de Alimentos no Brasil - um desafio político e social a ser vencido. Embrapa: Rio de Janeiro, 2014. Disponível em: $<$ http://atividaderural.com.br/artigos 1508fc56454d19.pdf>. Acesso em: 23 de maio de 2017.

6. VIZU, J.; GERVÁSIO, A.; ALVES, E.; SILVA, B. C. Aproveitamento do resíduo orgânico da casca de banana na elaboração de doces em pasta. In: Congresso Norte Nordeste de Pesquisa e Inovação, 7 , 2012, Palmas. Anais... Palmas: IFTO, 2012.

7. AIOLFI, A. H.; BASSO, C. Preparações elaborados com aproveitamento integral dos alimentos. Disciplinarum Scientia. Série: Ciências da Saúde, Santa Maria, v.14, n.1, p.109-114, 2013.

8. SERVIÇO SOCIAL DA INDÚSTRIA (SESI). Divisão de educação e cultura: guia de alimentação: missão possível 2: alimente-se bem infantojuvenil. São Paulo: SESI, 2013.

9. SILVA, M. C.; SILVA, T. J. A.; BONFIM-SILVA, E. M.; FARIAS, L. N. Características produtivas e qualitativas de melão rendilhado adubado com nitrogênio e potássio. Revista Brasileira de Engenharia Agrícola e Ambiental, Campina Grande, v.18, n.6, p.581-587, 2014.

10. RODRIGUES, R. B. 15 alimentos para melhorar a imunidade. In: RODRIGUES, R. B. Alimentação Saudável $=$ Saúde Perfeita. v.2. Joinville: Clube de autores, 2016 
11. SEBRAE NACIONAL. O cultivo e o mercado de melão. 2016. Disponível em: $<$ https://www.sebrae.com.br/sites/PortalSebrae/artigo s/o-cultivo-e-o-mercado-do-melao,5a 8837b644134410VgnVCM2000003c74010aRCRD>. Acesso em: 05 de abril de 2017.

12. CAVALCANTI, I. F. A.; FERREIRA, N. J.; DIAS, M. A. F. S.; SILVA, M. G. A. J. Tempo e clima no Brasil. São Paulo: Oficina de Textos, 2009.

13. TREICHEL, M. Anuário Brasileiro da Fruticultura. $2016 . \quad$ Disponível em: <http://www.grupogaz.com.br/tratadas/eo_edicao/4/2 016/04/20160414_0d40a2e2a/pdf/5149_2016fruticult ura.pdf>. Acesso em: 03 de maio de 2017.

14. GUERRA, A. G. Importância do cultivo de melão. In: GUERRA, A.G. Cultivo do melão - estufa. Natal: EMPARN, 2012.

15. PHILliPI, S. T. Tabela de composição de alimentos: suporte para decisão nutricional. 5 ed. Barueri: Manole, 2016.

16. CRUZ, C. O.; SANTOS, J. M.; CARDOZO, T. S. F. Aplicação do programa de educação nutricional: sexta é dia de fruta? É sim senhor!. Revista rede de cuidados em saúde, Duque de Caxias, v.3, n.2, p.112, 2014

17. PEREIRA, M. N.; SARMENTO, C. T. M. Oficina de culinária: uma ferramenta da educação nutricional aplicada na escola. Universitas: Ciências da Saúde, Brasília, v.10, n.2, p.87-94, 2012.

18. COSTA, M. G. F. A.; NUNES, M. M. J. C.; DUARTE, J. C.; PEREIRA, A. M. S. Conhecimento dos pais sobre alimentação: construção e validação de um questionário de alimentação infantil. Revista de Enfermagem Referência, Coimbra, v.3, n.6, p.55-68, 2012.

19. CARVALHO, K. H.; BOZATSKI, L. C.; SCORSIN, M.; NOVELLO, D.; PEREZ, E.; DALLA SANTA, H. S.; SCORSIN, G.; BATISTA, M. G. Desenvolvimento de cupcake adicionado de farinha da casca de banana: características sensoriais e químicas. Alimentos e Nutrição, Araraquara, v.23, n.3, p.475-481, 2012.

20. ASSOCIAÇÃO BRASILEIRA DAS INDÚSTRIAS DE BISCOITOS, MASSAS ALIMENTÍCIAS E PÃES E BOLOS INDUSTRIALIZADOS (ABIMAPI). Estatísticas de Pães \& Bolos. Disponível em: <http://www.abimapi.com.br/ estatistica-paes-bolos.php>. Acesso em: 05 de junho de 2017.

21. ORTOLAN, A. V.; EING, K. K. C.; SANTOS, M. M. R.; CANDIDO, C. J.; SANTOS, E. F.; NOVELLO, D. Adição de farinha de baru em cupcakes: caracterização físico-química e sensorial entre crianças. O Mundo da Saúde, São Paulo, v.40, n.2, p.213-220, 2016.

22. SILVA, A. R. M.; FERREIRA, K. F. B.; SOUSA, L. B.; ROLIM, N. A.; MONGE, S. M.; SILVA, V. P. Desenvolvimento de um novo produto: cupcake a base de banana e alfarroba isento de lactose. Revista Saber Científico, Porto Velho, v.5, n.1, p.52-60, 2016.

23. FONSECA, A. C. R. Marketing sensorial para crianças: A relação entre cor, sabor e textura. 2013, 51p. Dissertação (Mestrado em Marketing) Universidade da Beira Interior, Covilhã, 2013.

24. ANDRADE, T. F. Importância das análises físicoquímicas no controle de qualidade de alimentos consumidos em Santa Catarina. 2012. 32p. Monografia (Especialização em Saúde Pública) Universidade Federal de Santa Catarina, Florianópolis, 2012.

25. DUTCOSKY, S. D. Análise Sensorial de Alimentos. 3 ed. Curitiba: Champagnat, 2011.

26. AOAC INTERNATIONAL. Official Methods of Analysis of AOAC International. 18 ed. Gaithersburg: AOAC, 2011.

27. TABELA BRASILEIRA DE COMPOSIÇÃO DOS ALIMENTOS (TACO). Tabela Brasileira de Composição dos Alimentos. 4 ed. Campinas: NEPA, 2011.

28. MALLEK-AYADI, S.; BAHLOUL, N.; KECHAOU, N. Characterization, phenolic compounds and functional properties of Cucumis melo L. peels. Food Chemistry, Barking, v.221, n.1, p.1691-1697, 2016.

29. MERRILL, A. L.; WATT, B. K. Energy values of foods: basis and derivation. Agricultural Handbook. Washington: USDA, 1973.

30. DIETARY REFERENCE INTAKES (DRI). Dietary Reference Intakes for energy, carbohydrate, fiber, fat, fatty acids, cholesterol, protein and amino acids. Washington: National Academy Press, 2005.

31. MIRANDA, A. A.; CAIXETA, A. C. A.; FLÁVIO, E. F.; PINHO, L. Desenvolvimento e análise de bolos enriquecidos com farinha da casca do maracujá (passiflora edulis) como fonte de fibras. Alimentos e Nutrição, Araraquara, v.24, n.2, p.225-232, 2013.

32. MÄKILÄ, L.; LAAKSONEN, O.; KALLIO, H.; YANG, B. Effect of processing technologies and storage conditions on stability of black currant juices with special focus on phenolic compounds and sensory properties. Food Chemistry, Barking, v.221, n.1, p.422-430, 2016.

33. TADMOR, Y.; BURGER, J.; YAAKOV, Y.; FEDER, A.; LIBHABER, S. E.; PORTNOY, V.; MEIR, A.; TZURI, G.; SA'AR, U.; ROGACHEV, I.; AHARONI, A.; ABELIOVICH, H.; SCHAFFER, A. A.; LEWINSOHN, E.; KATZIR, N. Genetics of flavonoid, carotenoid, and chlorophyll pigments in melon fruit rinds. Journal of Agricultural and Food Chemistry, Washington, v.58, n.19, p.10722-10728, 2010.

34. CHEN. J.; ROSENTHAL, A. Woodhead Publishing Series in Food Science, Technology and Nutrition: Modifying Food Texture: Sensory Analysis, Consumer Requirements and Preferences. v.2. Reino Unido: Whoodhead Publishing, 2015.

35. COUTO, E. M. Utilização da farinha da casca de pequi (Caryocar brasiliense Camb.) na elaboração de pão de forma. 2007. 121p. Dissertação (Mestrado em Ciências dos Alimentos) - Universidade Federal de Lavras, Lavras, 2007.

36. TEIXEIRA. E.; MEINERT, E. M.; BARBETTA, P. A. Análise sensorial de alimentos. Florianópolis: Editora UFSC, 1987.

37. GASTALDON, L. T.; NOVELlO, D.; JUSTINO, FREITAS, A. R.; FRANCESCHINI, P. Análise sensorial de empadas integrais em crianças na fase escolar. Alimentos e Nutrição, Araraquara, v.18, n.3, p.303-307, 2007.

38. DALL'ALBA, V.; AZEVEDO, M. J. Papel das fibras alimentares sobre o controle glicêmico, perfil lipídicoe 
pressão arterial em pacientes com diabetes melito tipo 2. Revista Hospital de Clínicas de Porto Alegre, Porto Alegre, v.30, n.4, p.363-371, 2010.

39. AMARO, A. P.; BONILHA, P. R. M.; MONTEIRO, M. Efeito do tratamento térmico nas características físico-químicas e microbiológicas da polpa de maracujá. Alimentos e Nutrição, São Paulo, v.13, n.1, p.151-162, 2002.

40. ARAÚJO FILHO, D. G.; EIDAM, T.; BORSATO, A. V.; RAUPP, D. S. Processamento de produto farináceo a partir de beterrabas submetidas à secagem estacionária. Acta Scientiarum. Agronomy, Maringá, v.33, n.2, p.207-214, 2011.

41. MONDONI, J.; TOLEDO, N. M. V.; CANNIATTIBRAZACA, S. G. Caracterização físico-química e nutricional de farinha de cascas de melão. In: $11^{\circ}$ Simpósio Latino Americano de Ciência de Alimentos. Anais... Campinas: UNICAMP, 2015.

42. BERNAUD, F. S. R.; RODRIGUES, T. C. Fibra alimentar - ingestão adequada e efeitos sobre a saúde do metabolismo. Arquivos Brasileiros de Endocrinologia \& Metabologia, Porto Alegre, v.57, n.6, p.397-405, 2013.
43. BRASIL. Ministério da Saúde. Agência Nacional de Vigilância Sanitária (ANVISA). Resolução RDC n 263, de 22 de setembro de 2005. Regulamento Técnico para produtos de cereais, amidos, farinhas e farelos. Diário Oficial União; Poder Executivo, Brasília, DF, 2005.

44. QUAGLIA, G. Ciencia y tecnologia de la panificación. Zaragoza: Acribia, 1991.

45. GOESAERT, H.; BRIJS, K.; VERAVERBEKE, W. S.; COURTIN, C. M.; GEBRUERS, K.; DELCOUR, J. A. Wheat flour constituents: how they impact bread quality and how to impact their functionality. Trends in Food Science \& Technology, Cambridge, v.16, n.1, p.12-30, 2005.

46. JOOSTE, M. E.; MACKEY, A. O. Cake structure and palability as affected by emulsifying agents and baking temperatures. Journal of Food Science, Chicago, v.17, n.1-6, p.185-196, 1952.

47. BRASIL. Ministério da Saúde. Agência Nacional de Vigilância Sanitária (ANVISA). Resolução RDC n 54, de 12 de novembro de 2012. Regulamento técnico Mercosul sobre informação nutricional complementar (declarações de propriedades nutricionais). Diário Oficial União; Poder Executivo, Brasília, DF, 2012. 